\title{
ARTICLE Genetic and environmental factors strongly influence risk, severity and progression of age-related macular degeneration
}

\author{
Wenqiu Wang ${ }^{1}$, Katarzyna Gawlik ${ }^{2}$, Joe Lopez ${ }^{2}$, Cindy Wen ${ }^{1}$, Jie Zhu ${ }^{1}$, Frances Wu ${ }^{1}$, William Shi ${ }^{1}$, Samuel Scheibler ${ }^{1}$, Huimin Cai ${ }^{3,4}$,
} Ram Vairavan ${ }^{2}$, Alexander Shi ${ }^{1}$, Weldon Haw ${ }^{1,5}$, Henry Ferreyra ${ }^{1}$, Ming Zhang ${ }^{3}$, Sherman Chang ${ }^{2}$ and Kang Zhang ${ }^{1,3,5}$

Age-related macular degeneration (AMD) is characterized by complex interactions between genetic and environmental factors. Here we genotyped the selected 25 single-nucleotide polymorphisms (SNPs) in 983 cases with advanced AMD and 271 cases with intermediate AMD and build an AMD life-risk score model for assessment of progression from intermediate to advanced AMD. We analyzed the performance of the prediction model for geographic atrophy progressors or choroidal neovascularization progressors versus non-progressors based on the 25 SNPs plus body mass index and smoking status. Our results suggest that a class prediction algorithm can be used for the risk assessment of progression from intermediate to late AMD stages. The algorithm could also be potentially applied for therapeutic response, and toward personalized care and precision medicine.

Signal Transduction and Targeted Therapy (2016) 1, e16016; doi:10.1038/sigtrans.2016.16; published online 16 September 2016

\section{INTRODUCTION}

Age-related macular degeneration, an ocular degenerative disease, is regarded as the leading cause for irreversible vision loss in people age 55 or older in developed countries. ${ }^{1}$ The typical clinical signs of early AMD are drusen and retinal pigmental epithelium changes. The natural history of AMD is progressive, with gradual loss of visual function that may span over many years' time. In 10$15 \%$ of patients with dry AMD, the deterioration is more rapid and extensive and they suffer significant vision loss due to geographic atrophy (GA). In another approximately $10-15 \%$ of patients, the condition progresses to the 'wet' or neovascular form (also known as choroidal neovascularization, CNV). Approximately eight million people in the United States suffer symptoms of early or intermediate $A M D$, of whom almost one million will develop late AMD within the next 5 years. As aging population booms globally, AMD appears to be a significant public concern for the health care departments and scientific researchers. ${ }^{2,3}$

AMD, a heterogeneous and genetically complex disease, is triggered by multiple environmental and genetic risk factors., ${ }^{4,5}$ Epidemiological studies provided solid evidence that environmental factors including BMI (body mass index) and smoking increased risks of AMD. Our previous published study, as wells as other studies (such as AREDS studies), ${ }^{6-10}$ showed that several genetic variants linked strong association with AMD such as CFH, CFB, HTRA1/ARMS2 and others. A combined risk score including these multiple genetic loci along with demographic and environmental data was highly predictive of AMD phenotype. ${ }^{11-13}$ Although GA and CNV are regarded as late AMD suffering poor visual outcomes, GA and CNV require quite different treatment strategies accordingly due to their specific and different histopathological changes. Unfortunately, none of the reported assessment methods were able to precisely predict progression to the subtype of late AMD.
In the current study, we reported new prediction models for assessment of AMD development and disease progression based on 25 highly associated single-nucleotide polymorphisms (SNPs) from 15 genes (Supplementary Table 1) and 2 epidemiological factors. Each genetic or environmental risk factor was previously evaluated for association with AMD development and/or progression to different AMD stages. The lifetime risk model is capable to calculate the probability of developing AMD during the lifespan of an individual, although risk models for progression are providing the likelihood of progression from intermediate AMD stage to GA and CNV. We also proposed, based on the risk score distribution, the stratification approach that might provide a useful clinical tool.

\section{MATERIALS AND METHODS}

Study population

The study was approved by the institutional review boards of the University of California, San Diego, United states and West China Hospital, Chengdu, China. Informed written consent was obtained from each patient, and protocols were reviewed and approved by local ethics committees. All research adhered to the tenets of the Declaration of Helsinki. Between 2005 and 2010, patients were enrolled in a prospective study at Shiley Eye Institute and West China Hospital. All patients were examined by two experienced ophthalmologists and divided into three groups: (1) advanced AMD cases with geographic atrophy (anywhere within the grid and without any record of hemorrhage) or choroidal neovascularization (hemorrhagic retinal detachment, hemorrhage under the retina or retinal pigment epithelium, subretinal fibrosis) in the worse eye; (2) intermediate AMD cases with macular drusen (small, hard, large, large confluent, semigranual, cuticular, familial, soft confluent and soft noconfluent) $>63 \mu \mathrm{m}$ in the worse eye; (3) controls without known advanced or intermediate AMD. Smoking history was obtained at the baseline visit from questionnaires and participants were classified as current smokers, past smokers, or never smokers. BMI was derived from height and weight measurements at the baseline visit.

\footnotetext{
${ }^{1}$ Shiley Eye Institute and Institute for Genomic Medicine, San Diego, CA, USA; ${ }^{2}$ AutoGenomics Inc., Vista, CA, USA; ${ }^{3}$ Department of Ophthalmology and Molecular Medicine Research Center, West China Hospital, Sichuan University, Sichuan, China; ${ }^{4}$ Guangzhou KangRui Biological Pharmaceutical Technology Company, Guangzhou, China and ${ }^{5}$ Veterans Administration Healthcare System, San Diego, CA, USA.

Correspondence: M Zhang (zhangmingscu@126.com) or S Chang (schang@autogenomics.com) or K Zhang (kang.zhang@gmail.com)

Received 14 June 2016; revised 15 August 2016; accepted 16 August 2016
} 


\section{Genotyping}

Genomic DNA was extracted from peripheral blood leukocytes according to established protocols ${ }^{11}$ and genotyped by AutoGenomics using INFINITI High Throughput System (Vista, CA, USA) and AMD Panel (Vista, CA, USA, for research use only). On the INFINITI platform, dual levels of specificity are achieved by the multiplexing PCR and allele-specific primer extension (ASPE) processes. Target regions of relevant genes were amplified and the amplicons were served as templates for the ASPE, during which, fluorescentlabeled nucleotides were incorporated for signal amplification. Subsequently, ASPE primers were captured via hybridization of the primer's Tag-region with the anti-Tag oligonucleotides addressed on the BioFilmChip (Vista, CA, USA). The microarray was washed, dried and scanned for the data analysis using the INFINITI ACE Reader (Vista, CA, USA).

\section{Model building and internal validation}

Class prediction model building and testing were performed using TreeNet software (Salford Systems, San Diego, CA, USA). The binary logistic regression analysis with a 10 -fold cross-validation method was applied in the algorithm development. TreeNet (https://www.salford-systems.com/ products/TreeNet) randomly assigned 9/10th of the data (learning set) to the model building to compute the regression equation and thus establish the risk score model. Remaining 1/10th of the data were used for testing and served as the internal control. Receiver operating characteristic curve (ROC) was computed for both, the learning and testing sets. The closer ROC is between the learning and testing sets, the better class prediction algorithm is developed.

\section{Statistical analyses}

Statistical analyses were performed using SPSS 22.0 (SPSS, Chicago, IL, USA). All values are reported as the mean \pm s.d. or $95 \%$ confidence interval. $P$-values $<0.05$ were considered statistically significant.

\section{RESULTS}

Patient information

A total of 1677 unrelated individuals (957 Caucasians, 647 Asins, 39 Hispanics and 34 African Americans) were involved in this 7year study including 983 patients with advanced AMD (269 diagnosed with geographic atrophy and 714 with choroidal neovascularization), 271 patients with intermediate AMD, and 423 control patients. Patients with intermediate AMD were followed up for 7 years and did not progress to advanced stages, thus were considered non-progressors. Patients diagnosed with late AMD (geographic atrophy or choroidal neovascularization) were considered progressors. Table 1 shows the baseline characteristics of the study participants.

\section{SNPs selection and genotyping}

The following 15 loci with 25 common SNPs and established association with AMD were included in the present study to develop class prediction algorithms for lifetime risk assessment and disease progression: $A B C A 1$ (rs1883025); $A P O E$ (rs429358, rs7412); ARMS2 (rs10490924); C3 (rs2230199); CCDC109B (rs17440077); CETP (rs3764261); CFB (rs4151669, rs522162); CFH (rs1048663, rs1061170, rs10737680, rs1329428, rs2274700, rs3766405, rs412852); CFI (rs10033900); COL8A1 (rs13095226); HTRA1 (rs11200638); LIPC (rs493258, rs10468017); LPL (rs12678919); TIMP3 (rs9621532) and VEGFA (rs3025000, rs943080). A summary of the selected SNPs with the risk and non-risk alleles according to the literature, their affected genes and proposed roles in AMD pathogenesis is displayed in Supplementary Table 1.

We genotyped the selected 25 SNPs in 983 cases with advanced AMD, 271 cases with intermediate AMD, and 423 controls. We implemented stringent quality control criteria for each SNP in our data set. All variants showed high genotyping quality with an average call rate $>99.5 \%$.

\begin{tabular}{|c|c|c|c|c|}
\hline & \multirow{2}{*}{$\begin{array}{l}\text { Control } \\
n=423\end{array}$} & \multicolumn{3}{|c|}{$A M D$} \\
\hline & & $\begin{array}{c}C N V \\
(\mathrm{n}=714)\end{array}$ & $\begin{array}{c}G A \\
(\mathrm{n}=269)\end{array}$ & $\begin{array}{l}\text { Intermediate } \\
\quad(\mathrm{n}=271)\end{array}$ \\
\hline \multicolumn{5}{|l|}{ Age } \\
\hline media (range) & $68(49,96)$ & $77(44,97)$ & $81(49,101)$ & $82(47,107)$ \\
\hline \multicolumn{5}{|l|}{$B M I$} \\
\hline Mean \pm s.d. & $26.70 \pm 5.53$ & $26.47 \pm 5.21$ & $26.66 \pm 5.34$ & $26.02 \pm 5.16$ \\
\hline \multicolumn{5}{|l|}{$\operatorname{Sex}(n)$} \\
\hline Female & $223(52.7 \%)$ & 455 (63.7\%) & 161 (59.8\%) & 155 (57.2\%) \\
\hline Male & $200(47.3 \%)$ & 259 (36.3\%) & $108(40.2 \%)$ & 116 (42.8\%) \\
\hline \multicolumn{5}{|l|}{ Smoking (n) } \\
\hline Past & $143(33.8 \%)$ & 318 (44.5\%) & 79 (29.4\%) & 75 (27.7\%) \\
\hline Never & $256(60.5 \%)$ & 282 (39.5\%) & 102 (37.9\%) & 141 (52.0\%) \\
\hline Current & $17(4.0 \%)$ & $42(5.9 \%)$ & $8(3.0 \%)$ & $5(1.8 \%)$ \\
\hline NA & 7 (1.7\%) & 72 (10.1\%) & 80 (29.7\%) & 50 (18.5\%) \\
\hline
\end{tabular}

Abbreviations: AMD, age-related macular degeneration; $\mathrm{BMI}$, body mass index; CNV, choroidal neovascularization; GA, geographic atrophy; NA, data not available.

Risk prediction for developing AMD

We first assessed AMD lifetime risk defined as the likelihood of developing the disease during the individual's lifespan based only on genetic factors in our case-control study. We analyzed the performance of the prediction model in AMD patients (872 cases consisting of $269 \mathrm{GA}$ and $603 \mathrm{CNV}$ ) versus controls (423 cases) based on the 25 SNPs shown to be associated with the disease. The receiver operating characteristic curve (ROC) for the test set was 0.76 with $70 \%$ sensitivity and $66 \%$ specificity, where the positive (PPV) and negative (NPV) prediction values were $81 \%$ and $52 \%$, respectively (Figure 1 ).

To improve the performance of the model, we added non-genetic modifiable risk factors, BMl and smoking history (current, past or never). For this 27 variable model, the ROC for the test set was 0.79 with $70 \%$ sensitivity and $73 \%$ specificity, where PPV and NPV values were $84 \%$ and $54 \%$, respectively (Figures $2 a$ and b). The variable ranking showed the most important SNPs associated with AMD, along with non-genetic risk factors, at the top including $\mathrm{CFH}$ (rs412852), VEGFA (rs3025000), C3 (rs2230199) and ARMS2 (rs10490924), which is in accordance with previously published data (Figure $2 \mathrm{c}$ ). The AMD lifetime risk prediction model developed based on the 27 predictors (both genetic and non-genetic factors) was shown to be the most effective. The distribution of AMD lifetime risk score for cases and controls as observed in our study is given in Figure $3 a$. On the basis of the above results, the relative risk score of developing $\mathrm{AMD}$ can be generated and grouped into three risk categories: (1) low relative risk of $<35 \%$; (2) moderate relative risk of $35-67 \%$; and (3) high relative risk of $>67 \%$. These results suggest that a class prediction algorithm can be used for the risk assessment of developing AMD successfully.

Risk prediction for progression to geographic atrophy and choroidal neovascularization

We hypothesized that when applied to a group of patients with well-characterized intermediate AMD phenotype, a class prediction algorithm might also be used to predict disease progression. Progression was defined as transition from intermediate AMD to advanced AMD, either geographic atrophy (GA) or choroidal neovascularization (CNV), in the worse eye during a follow-up visit. 
a

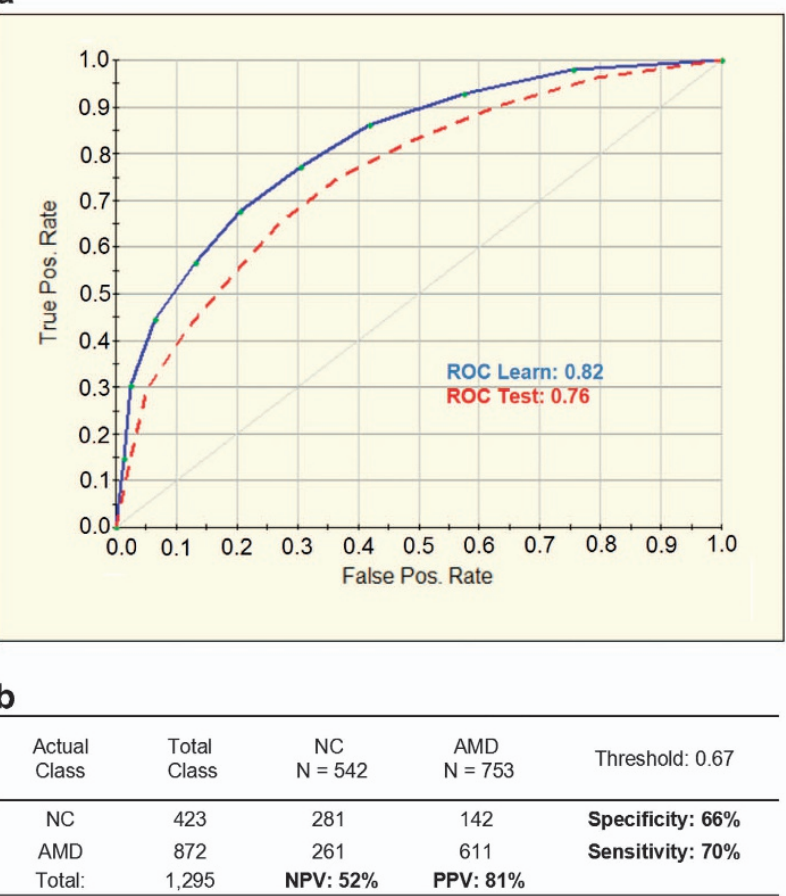

Figure 1. Performance of the model for AMD lifetime risk assessment based on 25 variables (genetic factors only). (a) Receiver operating characteristic curve (ROC) for the 25-SNP model was generated for learning (blue line) and testing (red dashed line) sets by using the binary logistic regression analysis with a 10-fold crossvalidation method. (b) Prediction success parameters were calculated for testing set. AMD, age-related macular degeneration cases; $\mathrm{NC}$, normal control; NPV, negative prediction value; PPV, positive prediction value.

We selected only patients with intermediate AMD who did not progress within 7 years to advanced AMD and considered them as non-progressors. Here we analyzed the performance of the prediction model for GA progressors (269 cases) or CNV progressors (714 cases) versus non-progressors (271 cases with intermediate AMD) based on the 27 variables ( 25 SNPs, BMI and smoking status). For both approaches we applied a 'shaving' technique, in which the predictors are ranked from top to bottom (the most important to the least important) at every step when the bottom predictor is removed and the model is rebuilt. This technique allowed us to choose the model for risk of progression to GA based on 10 variables with the best performance (Figure 4). The ROC for the test set was 0.71 with $67 \%$ sensitivity and $66 \%$ specificity, where PPV and NPV values were $66 \%$ and $67 \%$, respectively (Figures $4 a$ and b). The variable ranking (Figure $4 c$ ) showed only genetic factors at the top including APOE (rs7412), LPL (rs12678919), CFH (rs412852) and CCDC109B (rs17440077).

Using a 'shaving' technique for the modeling of progression risk to $\mathrm{CNV}$, we were not able to narrow down the number of the predictors and find an algorithm with better performance. Thus we evaluated the prediction model for risk of progression to CNV based on 27 variables (genetic and environmental factors). The ROC for the test set was 0.77 with $87 \%$ sensitivity and $62 \%$ specificity, where PPV and NPV values were $86 \%$ and $65 \%$, respectively (Figures $5 \mathrm{a}$ and b). The variable ranking (Figure $5 \mathrm{c}$ ) showed non-genetic risk factors at the top along with SNPs including ABCA1 (rs1883025), CETP (rs3764261), CFI (rs10033900) and $C F H$ (rs1048663). The distribution of GA and CNV progression risk score for progressors and non-progressors as observed in our study is given in Figures $3 \mathrm{~b}$ and $\mathrm{c}$. Our results suggest that a class prediction algorithm can also be used for the risk assessment of progression from intermediate to late AMD stages.

\section{DISCUSSION}

In the current study, we tested a panel of 25 SNPs for AMD association in 983 unrelated individuals diagnosed with late AMD, 271 patients with intermediate AMD and 423 controls. We demonstrated that multiple SNPs were sufficient to assess the AMD lifetime risk. Along with the non-genetic factors, smoking status and BMI, the algorithm was shown to be highly predictive and could differentiate between low, medium and high risk of developing AMD.

Before the first class prediction algorithm for AMD risk assessment was developed, we tested if a class prediction algorithm can be applied to categorical variables such as genotyping data. We found that a multivariate approach from the class prediction algorithm allowed the descriptive genotype data to be used directly for AMD risk prediction model building with comparable performance to the model built based on the numeric variables, thus eliminating the necessity of odds ratios calculations (Supplementary Figure 1).

AMD lifetime risk score is specifically developed to tell the likelihood of developing AMD during the lifespan of an individual. At first, we assessed our cases and controls based only on 25 genetic factors and the ROC was 0.76 with $70 \%$ sensitivity and $66 \%$ specificity. After adjusting for the smoking status and $\mathrm{BMI}$, the actual lifetime risk ROC increased to 0.79 with $70 \%$ sensitivity and $73 \%$ specificity, which indicated that smoking and BMI were involved as important AMD risk factors. According to our lifetime risk model, the relative score values of 8 variables: CFH (rs412852), BMI, VEGFA (rs3025000), SMOKING, C3 (rs2230199), ARMS2 (rs10490924), HTRA1 (rs11200638) and APOE (rs7412) were close to or $>50$, what proved their high contribution to the AMD development and reliability of the prediction model. Although there are no lifetime risk models reported elsewhere based exactly on the same combination of risk factors that were used here, they have been identified to have strong association with increased risk of AMD. ${ }^{11,14-23}$ The use of individual lifetime risk prediction might help to increased awareness of, and interest in, the importance and prevention of AMD in large population. They can also be used to guide the allocation of resources to improve public health services for AMD from both clinicians and the general public.

AMD is a slowly progressive retinal disease, and its course is variable and individual. In the current study, we defined progression as transition from intermediate AMD to advanced AMD, either geographic atrophy (GA) or choroidal neovascularization (CNV), in the worse eye during a follow-up visit. To identify whether association results differ between two subtypes of late AMD, we built two progression prediction models, respectively. In GA prediction model, 10 variables had been found to obtain the best performance and further evaluate with $67 \%$ sensitivity and $66 \%$ specificity. CNV prediction model was established based on 27 variables with $87 \%$ sensitivity and $62 \%$ specificity. Contrary to the CNV model, the GA model comprised none of the non-genetic factors, which suggests that genetic factors might have a predominant role in progression to $\mathrm{GA}$.

Surprisingly, in CNV model, BMI and smoking status showed larger contribution to CNV progression than genetic factors. BMI and smoking are the most important modifiable environmental risk factors associated with AMD. Obesity was shown to be associated with the incidence of AMD $\left(O R=1.04\right.$ per $\left.\mathrm{kg} \mathrm{m}^{-2}\right)$ in age, gene/environment susceptibility (AGES) study. Seddon, George et al. ${ }^{24}$ found that BMl of $25 \mathrm{~kg} \mathrm{~m}^{-2}$ or higher was found to increase the risk of advanced AMD for CT heterozygotes in CFH $\mathrm{Y} 402 \mathrm{H}$. For smoking, the risk of developing AMD was 3.7-fold higher in current smokers, and 1.8-fold higher in past smokers when compared with patients who never smoked. ${ }^{11,25}$ However, 
a

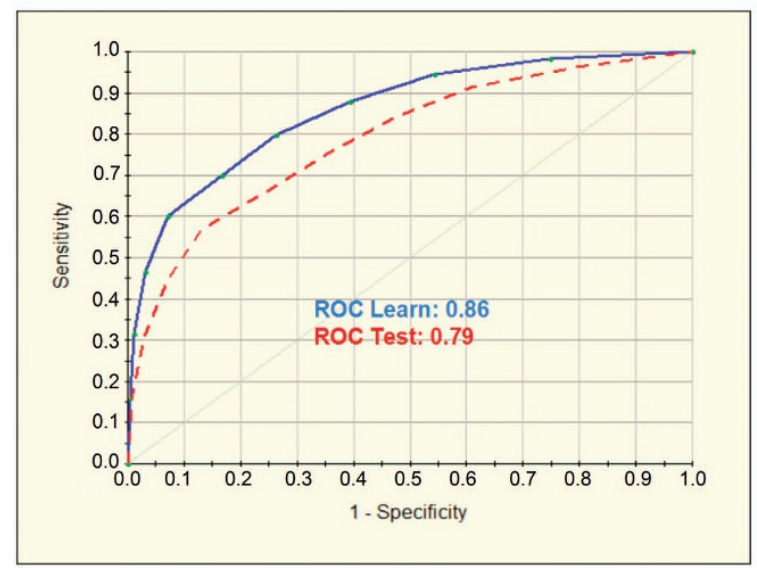

b

\begin{tabular}{ccccc}
\hline $\begin{array}{c}\text { Actual } \\
\text { Class }\end{array}$ & $\begin{array}{c}\text { Total } \\
\text { Class }\end{array}$ & $\begin{array}{c}\text { NC } \\
\mathrm{N}=568\end{array}$ & $\begin{array}{c}\text { AMD } \\
\mathrm{N}=727\end{array}$ & Threshold: 0.67 \\
\hline NC & 423 & 307 & 116 & Specificity: $\mathbf{7 3 \%}$ \\
AMD & 872 & 261 & 611 & Sensitivity: $\mathbf{7 0 \%}$ \\
Total: & 1,295 & NPV: $\mathbf{5 4 \%}$ & PPV: $\mathbf{8 4} \%$ & \\
\hline
\end{tabular}

c

\begin{tabular}{|l|l|l|}
\hline No & Variable & Score \\
\hline 1 & CFH (rs412852) & 100.00 \\
2 & BMI & 79.26 \\
3 & VEGFA (rs3025000) & 72.06 \\
4 & SMOKING & 71.52 \\
5 & C3 (rs2230199) & 59.43 \\
6 & ARMS2 (rs10490924) & 55.92 \\
7 & HTRA1 (rs11200638) & 52.78 \\
8 & APOE (rs7412) & 49.16 \\
9 & CFH (rs1061170) & 42.49 \\
10 & CFH (rs1329428) & 40.33 \\
11 & CFH (rs3766405) & 38.44 \\
12 & CFH (rs2274700) & 37.50 \\
13 & CFI (rs10033900) & 32.73 \\
14 & VEGFA (rs943080) & 32.67 \\
15 & LIPC (rs10468017) & 32.41 \\
16 & LIPC (rs493258) & 32.03 \\
17 & CCDC109B (rs17440077) & 30.64 \\
18 & CETP (rs3764261) & 30.38 \\
19 & APOE (rs429358) & 27.47 \\
20 & CFB (rs522162) & 25.98 \\
21 & ABCA1 (rs1883025) & 25.74 \\
22 & COL8A1(rs13095226) & 25.44 \\
23 & CFH (rs1048663) & 21.73 \\
24 & CFB (rs4151669) & 19.74 \\
25 & TIMP3 (rs9621532) & 16.43 \\
26 & CFH (rs10737680) & 16.15 \\
27 & LPL (rs12678919) & 16.13 \\
\hline
\end{tabular}

Figure 2. Performance of the model for AMD lifetime risk assessment based on 27 variables (genetic and environmental factors). (a) Receiver operating characteristic curve (ROC) for the 25-SNP plus smoking and BMI (body mass index) model was generated for learning (blue line) and testing (red dashed line) sets by using the binary logistic regression analysis with a 10 -fold cross-validation method. (b) Prediction success parameters were calculated for testing set. (c) Variable importance ranking is showing relative scores generated by TreeNet software and positioning the predictors from the most important to the least important. AMD, age-related macular degeneration cases; NC, normal control; NPV, negative prediction value; PPV, positive prediction value.

a

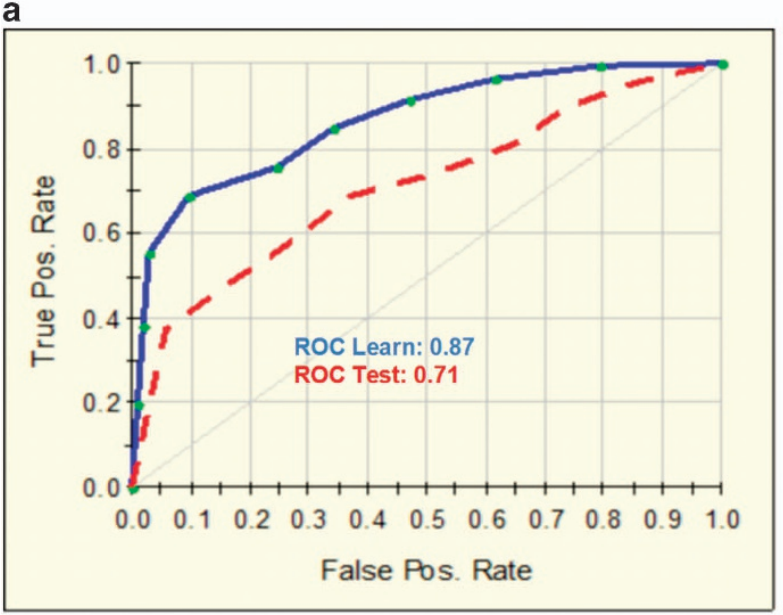

b

\begin{tabular}{ccccc}
\hline $\begin{array}{c}\text { Actual } \\
\text { Class }\end{array}$ & $\begin{array}{c}\text { Total } \\
\text { Class }\end{array}$ & $\begin{array}{c}\text { Intermediate } \\
\text { AMD } \\
\mathrm{N}=268\end{array}$ & $\begin{array}{c}\text { GA } \\
\mathrm{N}=272\end{array}$ & Threshold $=0.56$ \\
\hline $\begin{array}{c}\text { Intermediate } \\
\text { AMD }\end{array}$ & 271 & 179 & 92 & Specificity: $\mathbf{6 6 \%}$ \\
GA & 269 & 89 & 180 & Sensitivity: $\mathbf{6 7 \%}$ \\
Total: & 540 & NPV: $67 \%$ & PPV: $66 \%$ & \\
\hline
\end{tabular}

\section{c}

\begin{tabular}{|l|l|l|}
\hline No & Variable & Score \\
\hline 1 & APOE (rs7412) & 100.00 \\
2 & LPL (rs12678919) & 87.48 \\
3 & CFH (rs412852) & 76.10 \\
4 & CCDC109B (rs17440077) & 74.93 \\
5 & C3 (rs2230199) & 74.28 \\
6 & VEGFA (rs943080) & 74.17 \\
7 & LIPC (rs493258) & 71.82 \\
8 & CFI (rs10033900) & 70.52 \\
9 & VEGFA (rs3025000) & 68.88 \\
10 & CETP (rs3764261) & 68.03 \\
\hline
\end{tabular}

Figure 3. Distribution of the risk score computed in the prediction models. (a) AMD lifetime risk score distribution in the study population. AMD cases are shown in red and controls in blue. (b) GA progression risk score distribution in the study population. GA progressors are shown in brown and non-progressors in green. (c) CNV progression risk score distribution in the study population. CNV progressors are shown in purple and non-progressors in green. 
a

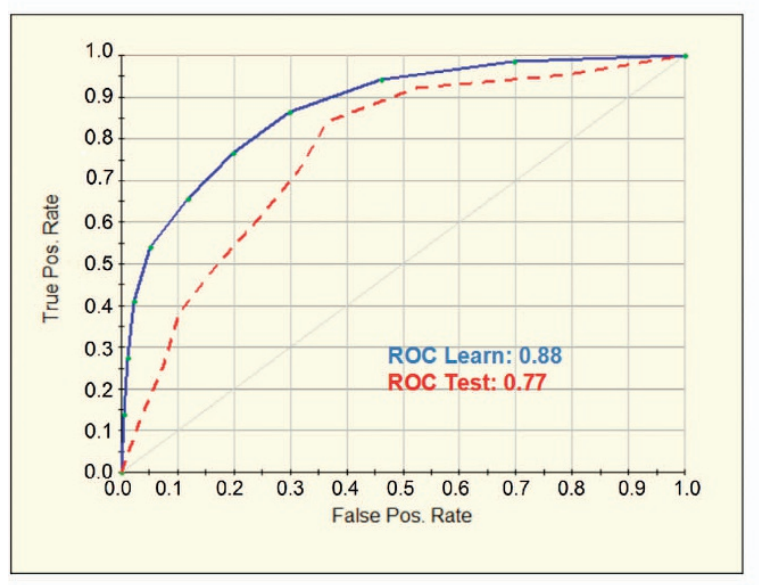

b

\begin{tabular}{ccccc}
\hline $\begin{array}{c}\text { Actual } \\
\text { Class }\end{array}$ & $\begin{array}{c}\text { Total } \\
\text { Class }\end{array}$ & $\begin{array}{c}\text { Intermediate } \\
\text { AMD } \\
\mathrm{N}=260\end{array}$ & $\begin{array}{c}\text { CNV } \\
\mathrm{N}=725\end{array}$ & Threshold: 0.72 \\
\hline $\begin{array}{c}\text { Intermediate } \\
\text { AMD }\end{array}$ & 271.00 & 169 & 102 & Specificity: $\mathbf{6 2 \%}$ \\
CNV & 714.00 & 91 & 623 & Sensitivity: $\mathbf{8 7 \%}$ \\
Total: & 985.00 & NPV: $\mathbf{6 5 \%}$ & PPV: $\mathbf{8 6 \%}$ & \\
\hline
\end{tabular}

\section{C}

\begin{tabular}{|l|l|l|}
\hline No & Variable & Score \\
\hline 1 & BMI & 100 \\
2 & SMOKING & 63.71 \\
3 & ABCA1 (rs1883025) & 28.55 \\
4 & CETP (rs3764261) & 28.53 \\
5 & CFI (rs10033900) & 26.73 \\
6 & CFH (rs1048663) & 20.37 \\
7 & VEGFA (rs3025000) & 20.3 \\
8 & COL8A1 (rs13095226) & 20.27 \\
9 & ARMS2 (rs10490924) & 20.08 \\
10 & APOE (rs7412) & 18.75 \\
11 & C3 (rs2230199) & 18.68 \\
12 & CFH (rs1061170) & 18.56 \\
13 & LIPC (rs10468017) & 17.22 \\
14 & CFH (rs3766405) & 17.15 \\
15 & APOE (rs429358) & 16.9 \\
16 & LIPC (rs493258) & 16.33 \\
17 & VEGFA (rs943080) & 16.16 \\
18 & LPL (rs12678919) & 15.99 \\
19 & HTRA1 (rs11200638) & 15.29 \\
20 & CCDC109B (rs17440077) & 15.15 \\
21 & CFH (rs412852) & 14.23 \\
22 & CFB (rs522162) & 13.99 \\
23 & TIMP3 (rs9621532) & 13.72 \\
24 & CFH (rs2274700) & 13.7 \\
25 & CFB (rs4151669) & 10.97 \\
26 & CFH (rs1329428) \\
27 & CFH (rs10737680) & 9.04 \\
& & 4.16 \\
\hline
\end{tabular}

Figure 4. Performance of the prediction model for 7-year progression to geographic atrophy (GA) based on 10 genetic variables. (a) Receiver operating characteristic curve (ROC) for the 10-SNP model was generated for learning (blue line) and testing (red dashed line) sets by using the binary logistic regression analysis with a 10-fold cross-validation method. (b) Prediction success parameters were calculated for testing set. (c) Variable importance ranking is showing relative scores generated by TreeNet software and positioning the predictors from the most important to the least important. NPV, negative prediction value; PPV, positive prediction value.

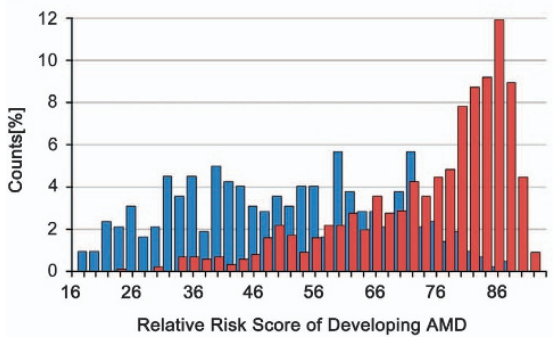

b

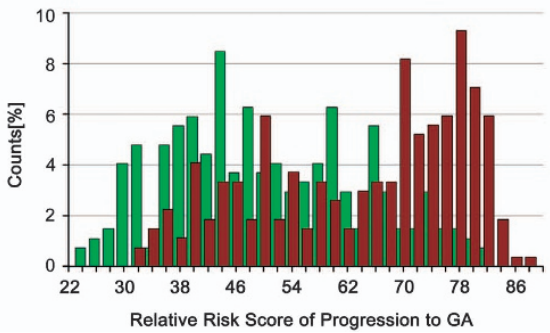

C

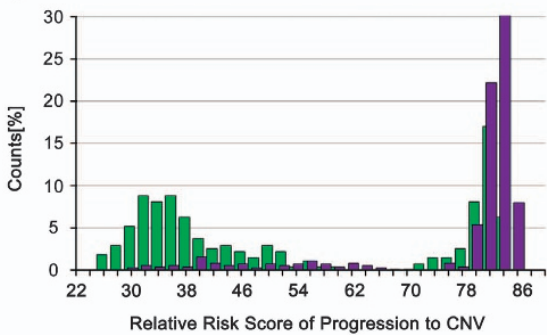

Figure 5. Performance of the prediction model for 7-year progression to choroidal neovascularization (CNV) based on 27 variables (genetic and environmental factors). (a) Receiver operating characteristic curve (ROC) for the 25-SNP plus smoking and BMI model was generated for learning (blue line) and testing (red dashed line) sets by using the binary logistic regression analysis with a 10-fold cross-validation method. (b) Prediction success parameters were calculated for testing set. (c) Variable importance ranking is showing relative scores generated by TreeNet software and positioning the predictors from the most important to the least important. NPV, negative prediction value; PPV, positive prediction value.

considered as self-estimated factors, weight control and smoking quitting were usually avoided to be assessed in most studies for CNV treatments. Although the way how the non-genetic factors acted on CNV course keeps unclear, our results provide an evidence for healthy lifestyle in preventing or slow-downing the progression of CNV.

Four SNPs, CFI (rs10033900), CETP (rs3764261), VEGFA (rs3025000) and APOE (rs7412), are found to overlap in top 10 variables of GA and CNV prediction. It may not necessarily reflect a shared etiology, but it may not necessarily reflect a shared etiology but may represent overlapping risk factors common to GA and CNV. On the other hand, it cannot be excluded that GA and CNV share a common etiology with slightly different endstage manifestation. In spite of these encouraging prediction results of individuals with CNV and GA, additional specific genegene and gene-environment interactions are still required to confirm these findings and to complement the relative weakness of the GA prediction.

The limitations of the presented models include the restriction of our analyses to mostly Caucasian population. The same polymorphism plays a different role in different ethnic populations or across different studies. The sample size for AMD risk prediction model is not large. We still need to be careful when extending the results to other populations. Nevertheless, we successfully built a prediction algorithm for risk of developing AMD and disease progression, and provided a strong basis for conducting future studies with much larger sample size to validate the initial findings. 


\section{ACKNOWLEDGEMENTS}

We thank the participants, their families and ophthalmologists who participated in this study. The study was partially supported by NSFC $(81300778,81271020), 973$ program (2012CB917304), 863 Program (2014AA021604), NIH (R01EY024134, R01HG008135, R01EY018660, R01EY021374) and VA Merit Award.

\section{AUTHOR CONTRIBUTIONS}

Involved in design of the study: WW, KG, MZ and KZ; collection of the data: $\mathrm{CW}$, $\mathrm{JZ}, \mathrm{FW}, \mathrm{WS}, \mathrm{MZ}$; analysis of the data: WW, KG, JL, SS, HC, RV, HF, SC; and interpretation of the data: WW, FW, KZ; and preparation of the manuscript: CW, $\mathrm{FW}$; revision of the manuscript WW, KG, SS, HC, SC; and approval of the manuscript: WW, KG, JL, CW, JZ, FW, WS, SS, HC, RV, HF, SC, KZ.

\section{COMPETING INTERESTS}

The authors declare no conflict of interest.

\section{REFERENCES}

1 Lim LS, Mitchell P, Seddon JM, Holz FG, Wong TY. Age-related macular degeneration. Lancet 2012; 379: 1728-1738.

2 Stevens GA, White RA, Flaxman SR, Price H, Jonas JB, Keeffe J et al. Global prevalence of vision impairment and blindness: magnitude and temporal trends, 1990-2010. Ophthalmology 2013; 120: 2377-2384.

3 Wong WL, Su X, Li X, Cheung CM, Klein R, Cheng CY et al. Global prevalence of age-related macular degeneration and disease burden projection for 2020 and 2040: a systematic review and meta-analysis. Lancet Glob Health 2014; 2: e106-e116.

4 Sobrin L, Seddon JM. Nature and nurture- genes and environment- predict onset and progression of macular degeneration. Progress Retin Eye Res 2014; 40: $1-15$.

5 Gopinath B, Flood VM, Kifley A, Liew G, Mitchell P. Smoking, antioxidant supplementation and dietary intakes among older adults with age-related macular degeneration over 10 years. PLOS ONE 2015; 10: e0122548.

6 Meyers KJ, Liu Z, Millen AE, lyengar SK, Blodi BA, Johnson E et al. Joint associations of diet, lifestyle, and genes with age-related macular degeneration. Ophthalmology 2015; 122: 2286-2294.

7 Clemons TE, Milton RC, Klein R, Seddon JM, Ferris FL 3rd. Age-Related Eye Disease Study Research Group. Risk factors for the incidence of Advanced Age-Related Macular Degeneration in the Age-Related Eye Disease Study (AREDS) AREDS report no. 19. Ophthalmology 2005; 112: 533-539.

8 Maller J, George S, Purcell S, Fagerness J, Altshuler D, Daly MJ et al. Common variation in three genes, including a noncoding variant in $\mathrm{CFH}$, strongly influences risk of age-related macular degeneration. Nat Genet 2006; 38: 1055-1059.

9 Yang Z, Camp NJ, Sun H, Tong Z, Gibbs D, Cameron DJ et al. A variant of the HTRA1 gene increases susceptibility to age-related macular degeneration. Science 2006; 314: 992-993.

10 Francis PJ, Hamon SC, Ott J, Weleber RG, Klein ML. Polymorphisms in C2, CFB and C3 are associated with progression to advanced age related macular degeneration associated with visual loss. J Med Genet 2009; 46: 300-307.

11 Chen Y, Zeng J, Zhao C, Wang K, Trood E, Buehler J et al. Assessing susceptibility to age-related macular degeneration with genetic markers and environmental factors. Arch Ophthalmol 2011; 129: 344-351.

12 Seddon JM, Reynolds R, Maller J, Fagerness JA, Daly MJ, Rosner B. Prediction model for prevalence and incidence of advanced age-related macular degeneration based on genetic, demographic, and environmental variables. Invest Ophthalmol Vis Sci 2009; 50: 2044-2053.

13 Seddon JM, Reynolds R, Yu Y, Daly MJ, Rosner B. Risk models for progression to advanced age-related macular degeneration using demographic, environmental, genetic, and ocular factors. Ophthalmology 2011; 118: 2203-2211.

14 Bergeron-Sawitzke J, Gold B, Olsh A, Schlotterbeck S, Lemon K, Visvanathan K et al. Multilocus analysis of age-related macular degeneration. Eur J Hum Genetics 2009; 17: 1190-1199.

15 Fritsche LG, Igl W, Bailey JN, Grassmann F, Sengupta S, Bragg-Gresham JL et al. A large genome-wide association study of age-related macular degeneration highlights contributions of rare and common variants. Nat Genet 2016; 48: 134-143.

16 Reynolds R, Hartnett ME, Atkinson JP, Giclas PC, Rosner B, Seddon JM. Plasma complement components and activation fragments: associations with age-related macular degeneration genotypes and phenotypes. Invest Ophthalmol Vis Sci 2009; 50: $5818-5827$

17 Yu Y, Bhangale TR, Fagerness J, Ripke S, Thorleifsson G, Tan PL et al. Common variants near FRK/COL10A1 and VEGFA are associated with advanced age-related macular degeneration. Hum Mol Genet 2011; 20: 3699-3709.

18 Chen W, Stambolian D, Edwards AO, Branham KE, Othman M, Jakobsdottir J et al. Genetic variants near TIMP3 and high-density lipoprotein-associated loci influence susceptibility to age-related macular degeneration. Proc Natl Acad Sci USA 2010; 107: 7401-7406.

19 Kaur I, Katta S, Reddy RK, Narayanan R, Mathai A, Majji AB et al. The involvement of complement factor B and complement component C2 in an Indian cohort with age-related macular degeneration. Invest Ophthalmol Vis Sci 2010; 51: 59-63.

20 Naj AC, Scott WK, Courtenay MD, Cade WH, Schwartz SG, Kovach JL et al. Genetic factors in nonsmokers with age-related macular degeneration revealed through genome-wide gene-environment interaction analysis. Ann Hum Genet 2013; 77: 215-231.

21 Neale BM, Fagerness J, Reynolds R, Sobrin L, Parker M, Raychaudhuri S et al. Genome-wide association study of advanced age-related macular degeneration identifies a role of the hepatic lipase gene (LIPC). Proc Natl Acad Sci USA 2010; 107: 7395-7400.

22 Abedi F, Wickremasinghe S, Richardson AJ, Makalic E, Schmidt DF, Sandhu SS et al. Variants in the VEGFA gene and treatment outcome after anti-VEGF treatment for neovascular age-related macular degeneration. Ophthalmology 2013; 120: $115-121$.

23 Fritsche LG, Chen W, Schu M, Yaspan BL, Yu Y, Thorleifsson G et al. Seven new loci associated with age-related macular degeneration. Nat Genet 2013; 45: 433-439, e1-e2.

24 Seddon JM, George S, Rosner B, Klein ML. CFH gene variant, $\mathrm{Y} 402 \mathrm{H}$, and smoking, body mass index, environmental associations with advanced age-related macular degeneration. Hum Hered 2006; 61: 157-165.

25 Ni Dhubhghaill SS, Cahill MT, Campbell M, Cassidy L, Humphries MM, Humphries P. The pathophysiology of cigarette smoking and age-related macular degeneration. Adv Exp Med Biol 2010; 664: 437-446.

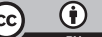

This work is licensed under a Creative Commons Attribution 4.0 International License. The images or other third party material in this article are included in the article's Creative Commons license, unless indicated otherwise in the credit line; if the material is not included under the Creative Commons license, users will need to obtain permission from the license holder to reproduce the material. To view a copy of this license, visit http://creativecommons.org/licenses/ by/4.0/

(c) The Author(s) 2016

Supplementary Information accompanies the paper on the Signal Transduction and Targeted Therapy website (http://www.nature.com/sigtrans) 